\title{
Clusters of Orthologous Groups of Genes
}

National Cancer Institute

\section{Source}

National Cancer Institute. Clusters of Orthologous Groups of Genes. NCI Thesaurus. Code C19640.

Each COG consists of individual orthologous proteins or orthologous sets of paralogs from at least three lineages. Orthologs typically have the same function, allowing transfer of functional information from one member to an entire COG. (from Science $1997 ; 278: 631-7)$ 